\title{
Metanálise para características de carcaça de bovinos de diferentes grupos genéticos
}

\author{
Metanalysis for carcass traits of cattle from different genetic groups
}

\author{
Jader Silva Lopes ${ }^{I}$ Paulo Roberto Nogara Rorato ${ }^{\text {II }}$ Tomás Weber ${ }^{\mathrm{I}}$ Rogério Dias Rodrigues ${ }^{\text {III }}$ \\ Juliana Grigoletto Comin ${ }^{\text {III }}$ Mariana de Almeida Dornelles ${ }^{\text {III }}$
}

\section{RESUMO}

A fim de avaliar os fatores que afetam os componentes da qualidade da carcaça em bovinos (área de olho de lombo - AOL e espessura de gordura de cobertura entre a $12^{a}$ e a $13^{a}$ costela - EGC) e compará-los nos diferentes grupos genéticos foi aplicada a metodologia da metanálise. Foram estudadas 215 estimativas de AOL e 209 de EGC, obtidas de 65.174 animais, extraídas de 36 artigos publicados entre 1985 e 2006. Aplicou-se análise de variância pelo método da Máxima Verossimilhança Restrita sob o modelo que explicava a variação das características em função das variações do país de origem, da categoria animal, do sistema de alimentação, metodologia de mensuração, contribuição dos genes zebuínos, taurinos britânicos e taurinos continentais, idade e do peso à mensuração. As médias observadas para as características AOL e EGC foram, respectivamente, $63,5 \mathrm{~cm}^{2}$ e 4,2 $\mathrm{mm}$. Os animais tinham, em média, 552,22 dias de vida à mensuração dos atributos e pesavam $468,47 \mathrm{~kg}$ de peso vivo. O uso da metanálise permitiu conclusões mais generalizadas a respeito da AOL e EGC: animais criados nos EUA apresentaram valores significativamente superiores para AOL e EGC quando comparados àqueles criados na Austrália e no Brasil; machos, inteiros e castrados apresentaram maiores medidas de AOL, não diferindo significativamente entre si, do que as fêmeas, sendo que as maiores EGC foram observadas nas fêmeas. Os sistemas de alimentação, confinado e semiconfinado, foram superiores ao sistema a campo. Os genótipos taurinos continentais apresentaram os maiores valores de AOL, já os taurinos britânicos apresentaram os maiores valores de EGC, sendo que os zebuínos apresentaram valores intermediários. Não houve diferença entre as medidas realizadas na carcaça (pós-morten) e por ultra-som (in vivo). As características apresentaram acréscimos decrescentes à medida que a idade e o peso vivo aumentaram.
Palavras-chave: área de olho de lombo, espessura de gordura de cobertura, taurinos britânicos, taurinos continentais, zebuínos.

\section{ABSTRACT}

The meta-analysis methodology was applied in this study to evaluate factors that affect the carcass quality components in beef cattle (rib eye area - AOL and fat thickness - EGC, measured between the $12^{\text {th }}$ and the $13^{\text {th }}$ ribs) and to compare them in different genetic groups. Data from 215 measures of AOL and 209 measures of EGC, from 65,174 animals, extracted from 36 papers published between 1985 and 2006 were used. The Restricted Maximum Likelihood Method and an animal model that considered AOL and EGC as functions of the fixed effects of country from where the data come from; animal category; feed system; measure methodology; Bos indicus, british and continental Bos taurus gene contribution; age and weight at measure, were used. The observed averages for AOL and e EGC were $63.5 \mathrm{~cm}^{2}$ and $4.2 \mathrm{~mm}$, respectively. The animals were in average 552.22 days old at the measure of the characteristics and had $468.47 \mathrm{~kg}$ of live weight. The use of meta-analysis permitted to get generalized conclusions about AOL and EGC: animals rose in the EUA presented values significantly superior for AOL and EGC when compared with those rose in Australia and Brazil; castrated and the entire males presented bigger AOL measures (do not differing among them) compared to females, and females presented bigger EGC. The feed system, confined and do not confined, were superior to the greasing system. The Continental Bos taurus genotypes presented bigger AOL values, British Bos taurus presented bigger EGC values, and Bos indicus presented intermediate values. The measures taken in the carcass (pos mortis) and by ultra son (in vivo) were not different. The increase in the characteristics decreased with the age and live weight increase.

'Programa de Pós-graduação em Zootecnia, Departamento de Zootecnia, Universidade Federal de Santa Maria (UFSM), Santa Maria, RS, Brasil.

IIDepartamento de Zootecnia, UFSM, 97105-970, Santa Maria, RS, Brasil. E-mail: rorato@smail.ufsm.br. Autor para correspondência.

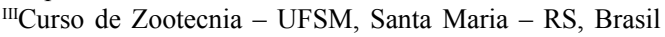


Key words: British and Continental Bos taurus, Bos indicus, fat thickness, rib eye area.

\section{INTRODUÇÃO}

A bovinocultura de corte vem se tornando mais competitiva a cada ano, reduzindo as margens de lucro e fazendo com que os produtores lancem mão das tecnologias disponíveis visando o aumento da produtividade. Segundo CATTANI (2006), a população mundial passou de 2,5 bilhões em 1950 para 6,8 bilhões de habitantes em 2005, enquanto que a área cultivada aumentou apenas em 10 milhões de hectares (ha), reduzindo a proporção superfície cultivada/habitante de 0,56ha para 0,22ha. Esta redução explica a necessidade de maior eficiência no uso de recursos caros e agora escassos como a terra, elevando a produtividade animal por ha, sem alterar a sustentabilidade da produção. No que se refere à pecuária, o conhecimento das potencialidades e limitações das diferentes raças é importante, pois fornece orientação para programas de cruzamento voltados ao aproveitamento dos benefícios da heterose, da combinação aditiva e da complementaridade entre as raças, com o objetivo final de promover a melhoria na eficiência e na qualidade do produto.

O cruzamento entre raças européias e zebuínas vem sendo utilizado amplamente em nosso meio criatório, com o objetivo de produzir animais com maior desempenho nas condições de ambiente em que são explorados. Esta é uma prática habitual em países como Estados Unidos da América (EUA), Canadá, Nova Zelândia e na zona temperada da Austrália, onde 80 a $90 \%$ dos bovinocultores de corte a utilizam (SUNDSTROM et al., 1994), e no Uruguai, com $40 \%$ das propriedades fazendo uso dos cruzamentos entre raças (AGUILAR \& BRIZOLARA, 1995).

A utilização das raças européias (Bos taurus

taurus) britânicas em cruzamentos é feita no intuito de aumentar a qualidade da carne dos produtos, além de aumentar a precocidade, tanto de crescimento/ acabamento quanto reprodutiva. As raças zebuínas, em geral, são mais produtivas do que as taurinas em climas tropicais devido a sua maior resistência a parasitas e à tolerância ao calor. Todavia, segundo $\mathrm{KOCH}$ et al. (1982), WHEELER et al. (1990), GALLINGER et al. (1992), LEME et al. (2000) e SILVA et al. (2003), apresentam qualidade de carcaça inferior em relação às taurinas britânicas. Entretanto, quando utilizadas em cruzamentos com raças taurinas, maximizam o efeito de heterose. Já as raças européias continentais apresentam alta taxa de crescimento, além de elevados pesos a maturidade, sendo consideradas raças terminais em cruzamentos.

Estudos comparando carcaças de diferentes genótipos, puros e/ou cruzados, não são raros (CROUSE et al., 1989; GREGORY et al., 1994; MARSHALL, 1994; BIDNER et al., 2002; CASAS \& CUNDIFF, 2003; SANCHEZ et al., 2005; entre outros). Entretanto, a variabilidade existente entre os estudos, que pode ser proveniente, por exemplo, de diferentes tipos de manejo alimentar, diferentes categorias, sexos, métodos de avaliação, dados oriundos de vários países, idades ao abate distintas, diferentes números de $\operatorname{amostras}(n)$, etc. impede que sejam feitas comparações conclusivas entre as qualidades das carcaças dos diferentes genótipos. Assim, um procedimento estatístico que propõe estimar e, se necessário, incluir na análise conjunta a variância entre os estudos é a metaanálise, metodologia que combina os resultados de vários experimentos que examinam o mesmo tema com o objetivo de sumarizar um conjunto de evidências (HAUPTLI et al., 2007), acarretando em alguma conclusão geral sobre o tema em estudo. Ela foi realizada pioneiramente por Karl Pearson em 1904, para resolver o problema da precisão reduzida de estudos com amostras ( $n$ ) pequenas (HEDGES \& OLKIN, 1985).

O objetivo deste estudo foi aplicar a metodologia da metanálise para avaliar os fatores que afetam componentes da qualidade da carcaça de bovinos, tais como a área de olho de lombo e a espessura de gordura de cobertura, medidas entre a $12^{\mathrm{a}}$ e a $13^{\mathrm{a}}$ costelas, em diferentes grupos genéticos.

\section{MATERIAL E MÉTODOS}

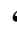

Foram utilizados neste estudo dados referentes a 215 estimativas de área de olho de lombo (AOL) e 209 estimativas de espessura de gordura de cobertura entre a $12^{\mathrm{a}}$ e a $13^{\mathrm{a}}$ costela (EGC), oriundas de 36 artigos publicados entre 1985 e 2006 e de 65.174 bovinos. Estes artigos foram acessados nos portais "CAPES", "PubMed" e "SciELO". A princípio foram encontrados 53 artigos que tratavam do estudo de área de olho de lombo e/ou espessura de gordura de cobertura em carcaças de bovinos, entretanto, 17 destes não apresentavam alguma informação importante para a aplicação da metaanálise em questão, como informação da idade ou do peso do animal à mensuração das características e assim não foram utilizados para o estudo.

A metanálise constituiu-se dos seguintes passos:

- análise exploratória conjunta dos dados: visa detectar tanto a presença de vício de publicação, isto é, se a 
pesquisa foi bastante abrangente, quanto a presença de observações discrepantes, além de fazer uma análise exploratória inicial dos dados por meio de estatísticas univariadas. $\mathrm{O}$ vício de publicação foi examinado por meio do gráfico de funil, que consiste em um diagrama de dispersão das estimativas de AOL e EGC contra o tamanho da amostra $(n)$. É baseado no fato da i-ésima estimativa de AOL ou EGC ser tanto mais precisa quanto maior o $n$, e de estudos com menores $n$ serem prevalecentes na literatura. $\mathrm{Na}$ ausência de vício de publicação, a forma do gráfico será semelhante a um funil invertido e simétrico (EGGER \& SMITH, 1998); - realização do teste de homogeneidade: a validação dos procedimentos utilizados na metaanálise requer que as suposições de normalidade e independência das estimativas a serem combinadas sejam satisfeitas (HEDGES \& OLKIN, 1985). A independência das estimativas é em parte garantida, em virtude de essas serem obtidas de diferentes trabalhos publicados, já a normalidade deve ser testada. O teste de Shapiro-Wilk foi utilizado para verificar a suposição de normalidade (SHAPIRO \& WILK, 1965) por meio do procedimento UNIVARIATE (SAS, 2001);

- identificação e mensuração dos fatores que afetam significativamente as características AOL e EGC: na presença da normalidade foi realizado estudo de análise de variância pelo método da Máxima Verossimilhança Restrita por meio do procedimento MIXED (SAS, 2001), conforme sugerido por ST-PIERRE (2007), usando o modelo:

$\mathrm{Y}_{\mathrm{jklmn}}=\mu+\mathrm{P}_{\mathrm{j}}+\mathrm{Ca}_{\mathrm{k}}+\mathrm{A}_{1}+\mathrm{M}_{\mathrm{m}}+\beta_{1} \mathrm{Z}_{\mathrm{jklm}}+\beta_{2} \mathrm{~B}_{\mathrm{jklm}}+$ $\beta_{3} \mathrm{Co}_{\mathrm{jklm}}+\beta_{4} \mathrm{Id}_{\mathrm{jklm}}+\beta_{5} \mathrm{PV}_{\mathrm{jklm}}+\varepsilon_{\mathrm{jklmn}}$

em que: $Y_{j k l m n}=$ variável dependente ponderada (AOL, EGC); $\mu$ = média geral; $\mathrm{P}_{\mathrm{j}}=$ efeito do j-ésimo país (Austrália, Brasil ou Estados Unidos da América - EUA); $\mathrm{Ca}_{\mathrm{k}}=$ efeito da k-ésima categoria animal (novilhos inteiros, castrados, novilhas ou vacas de descarte); $\mathrm{A}_{1}=$ efeito do l-ésimo tipo de alimentação (campo, confinado ou semi-confinado); $\mathrm{M}_{\mathrm{m}}=$ efeito da m-ésima metodologia de mensuração (ultra-som ou pós-mortis); $\mathrm{Z}_{\mathrm{jklm}}=$ efeito do genótipo zebuíno; $\mathrm{B}_{\mathrm{jklm}}$ $=$ efeito do genótipo europeu britânico; $\mathrm{Co}_{\mathrm{jklm}}=$ efeito do genótipo europeu continental; $\mathrm{Id}_{\mathrm{jklm}}=$ efeito da covariável idade do animal à mensuração, efeitos linear e quadrático; $\mathrm{PV}_{\mathrm{jklm}}=$ efeito da covariável peso vivo do animal à mensuração, efeitos linear e quadrático; $\varepsilon_{\mathrm{jklmn}}=$ erro aleatório associado a $n$-ésima observação; e $\beta_{1}, \beta_{2}, \beta_{3} \beta_{4}$ e $\beta_{5}=$ coeficientes de regressão. $O$ fator de ponderação foi o tamanho da amostra que dera origem a cada estimativa de AOL ou EGC.

\section{RESULTADOS E DISCUSSÃO}

As médias observadas para as características AOL e EGC foram, respectivamente, $63,5 \mathrm{~cm}^{2}$ e 4,2mm. Os animais tinham, em média, 552,22 dias de vida à mensuração dos atributos e pesavam $468,47 \mathrm{~kg}$ de peso vivo, com um rendimento médio de carcaça de $57,86 \%$ para os animais que tiveram suas mensurações feitas no pós-mortem.

As contribuições dos genótipos variaram de zero a um, com os animais tendo em média 0,28; 0,41 e 0,26 do genótipo zebuíno, europeu britânico e continental, respectivamente.

Os gráficos de funil (Figura 1) mostram que não houve vício de publicação na pesquisa bibliográfica realizada para este estudo, já que os gráficos apresentam forma semelhante a de um funil invertido, com tamanhos de amostras concentradas entre 25 e 200 animais.

A partir dos testes de Shapiro-Wilk, cuja hipótese de nulidade expressa que os dados em questão têm distribuição normal, pôde-se verificar a existência de normalidade na distribuição dos dados de AOL e EGC, sendo que as estatísticas W de ShapiroWilk foram 0,97 $(\mathrm{P}=0,44)$ e $0,94(\mathrm{P}=0,31)$, para AOL e EGC, respectivamente.

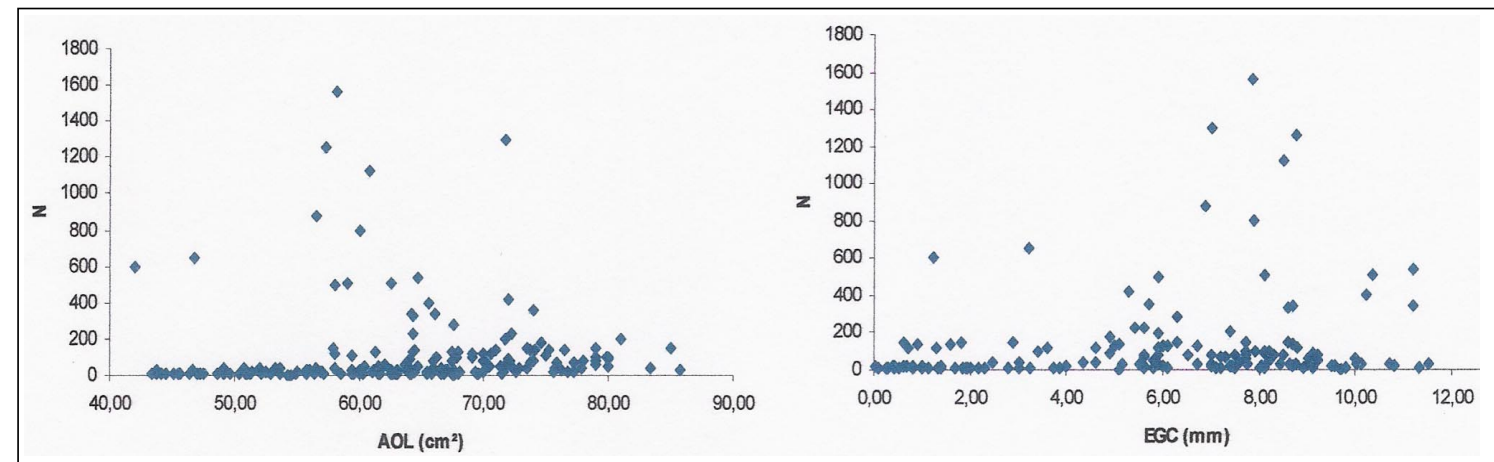

Figura 1 - Gráficos de funil das medidas de área de olho de lombo (AOL) e espessura de gordura de cobertura (EGC), relacionadas ao tamanho da amostra $(\mathrm{N})$ que lhes dera origem. 
Com as suposições de normalidade e independência das estimativas confirmadas, foi realizada a análise de variâncias das características. Os resultados das comparações entre os efeitos de classe são apresentados na tabela 1 . O modelo utilizado explicou $82,53 \%$ da variação da AOL e $79,04 \%$ da variação da EGC, com coeficientes de variação de 7,51\% e $12,33 \%$ para AOL e EGC, respectivamente.

Os animais criados nos EUA tiveram valores significativamente diferentes e superiores para AOL e EGC quando comparados àqueles criados na Austrália e no Brasil. A média de EGC para o Brasil foi de 4,07mm, valor este considerado aquém do desejado, recomendando-se no mínimo 5,00mm de EGC para que o animal seja abatido com um bom acabamento de carcaça, evitando problemas de qualidade de carcaça e perdas ao resfriamento (MÜLLER, 1987; USDA, 1997).

Quando foram comparadas as categorias animais, os machos, inteiros e castrados, apresentaram maiores medidas de AOL, não diferindo significativamente entre si. Isto se deve aos mais elevados níveis do hormônio testosterona nos machos, comparados às fêmeas, o qual aumenta a retenção de líquidos, aumentando o volume muscular (CUNNINGHAM, 2004). Por outro lado, nas fêmeas foram observadas as maiores EGC, o que é explicado pelo fato destas possuírem menores pesos e idades à maturação (GARNERO et al., 2005). Desse modo, elas, são mais precoces na deposição de gordura e em

Tabela 1 - Médias, ajustadas para o modelo, para área de olho de lombo (AOL) e espessura de gordura de cobertura (EGC), segundo os diferentes efeitos de classe.

\begin{tabular}{|c|c|c|c|}
\hline & Efeitos & $\operatorname{AOL}\left(\mathrm{cm}^{2}\right)$ & $\mathrm{EGC}(\mathrm{mm})$ \\
\hline \multirow{3}{*}{ País } & Austrália & $62,86 \mathrm{~b}$ & $6,61 b$ \\
\hline & Brasil & $62,77 \mathrm{~b}$ & $4,07 \mathrm{c}$ \\
\hline & EUA & $66,37 \mathrm{a}$ & $9,19 \mathrm{a}$ \\
\hline \multirow{4}{*}{ Categoria } & Macho inteiro & $67,31 \mathrm{a}$ & $4,05 \mathrm{c}$ \\
\hline & Macho castrado & $65,13 \mathrm{a}$ & $6,61 b$ \\
\hline & Novilha & $59,79 \mathrm{c}$ & $7,28 \mathrm{a}$ \\
\hline & Vaca de descarte & $61,02 b$ & $7,55 \mathrm{a}$ \\
\hline \multirow{3}{*}{ Alimentação } & Confinado & $72,18 \mathrm{a}$ & $8,43 \mathrm{a}$ \\
\hline & Semi-confinado & $71,94 a$ & $6,29 \mathrm{ab}$ \\
\hline & Campo & $62,14 \mathrm{~b}$ & $3,14 \mathrm{~b}$ \\
\hline \multirow{2}{*}{ Metodologia } & Ultra-som & 62,81 & 3,96 \\
\hline & Pós-morte & 64,69 & 5,62 \\
\hline
\end{tabular}

EUA - Estados Unidos da América.

Médias seguidas de mesma letra na coluna não diferem a 5\% de significância pelo teste Least Squares Means (SAS, 2001). acabamento de carcaça. Estes valores concordam com URICK et al. (1991), os quais compararam novilhos e novilhas de diferentes composições genéticas.

Embora com níveis distintos de suplementação, os sistemas de alimentação confinado (todo o alimento fornecido no cocho) e semi-confinado (parte do alimento fornecido adicionalmente ao pastejo) mostraram resultados semelhantes quanto às características estudadas. Estes foram superiores ao sistema campo, em que a única forma de suplementação era a mineral. O maior aporte de nutrientes, elevando a qualidade da dieta com a suplementação ou o confinamento, pode ser uma estratégia para se atingir níveis adequados de acabamento de carcaça, elevar o rendimento de carcaça e a proporção de cortes nobres nesta (relacionados à $\mathrm{AOL}$ ) ou ainda reduzir o tempo necessário para isso (RESTLE et al., 2003).

A determinação de características de carcaça de maneira rápida, com boa acurácia e, principalmente, não invasiva é uma necessidade que pode ser obtida por ultra-sonografia (ANDERSEN et al., 1983). Tal afirmativa foi corroborada pelo presente estudo, o qual apontou a inexistência de diferença entre as medidas realizadas na carcaça (pós-mortem) e aquelas realizadas por ultra-som (in vivo). Na literatura não é raro encontrar autores que evidenciaram alta correlação entre a espessura da gordura subcutânea, a área do músculo Longissimus dorsi e a composição da carcaça, medidas por ultra-som no animal vivo, com as mesmas medidas na carcaça (HEDRICK, 1983; ROUSE et al., 1992; SILVA et al., 2003). Entretanto, RIBEIRO et al. (1999a; 1999b) relataram correlações mais baixas entre essas medidas, o que pode estar relacionado com as limitações tecnológicas, a experiência do técnico, o nível de gordura e de músculo, o sexo e a idade do animal, as mudanças nas características dos tecidos pós-morte, a remoção da gordura junto com o couro e o deslocamento dos músculos em relação ao esqueleto, sendo estes os principais fatores que interferem na acurácia das medidas feitas com o ultra-som e daquelas feitas diretamente na carcaça (PERKINS, 1992).

As equações que descrevem a variação das características AOL e EGC, corrigidas para os efeitos de classe, em função dos efeitos contínuos são: $\mathrm{AOL}_{\mathrm{aj}}=-28,46-4,27 \mathrm{Z}-3,65 \mathrm{~B}+0,34 \mathrm{Co}+0,13 \mathrm{Id}-$ $68.10^{-6} \mathrm{Id}^{2}+0,12 \mathrm{PV}-47.10^{-6} \mathrm{PV}^{2}$

$\mathrm{EGC}_{\mathrm{aj}}=-28,56-2,83 \mathrm{Z}+0,59 \mathrm{~B}-3,80 \mathrm{Co}+0,04 \mathrm{Id}-$ $19.10^{-6} \mathrm{Id}^{2}+0,05 \mathrm{PV}-25.10^{-6} \mathrm{PV}^{2}$

As características AOL e EGC apresentaram comportamento semelhante com relação à variação da idade e do peso vivo à mensuração, mostrando acréscimos decrescentes à medida que a idade e o peso vivo aumentaram, dentro do intervalo de estudo, ou 
seja, os maiores acréscimos foram observados em animais mais jovens. Resultados semelhantes foram encontrados por SILVA et al. (2003) para novilhos da raça Nelore no Brasil. Por outro lado, este comportamento de aumentos acentuados na AOL será evidenciado até aproximadamente os cinco anos de idade, dependendo da raça, quando essa medida tende a se estabilizar com a chegada do peso assintótico dos bovinos.

A partir dos coeficientes de regressão dos grupos genéticos pode-se inferir que os genótipos taurinos continentais possuem as maiores AOL e que seu uso em cruzamentos eleva o rendimento de carcaça dos produtos. Da mesma forma, GREGORY et al. (1994) encontraram maiores valores para AOL em animais taurinos continentais comparados aos taurinos britânicos e BIDNER et al. (2002), introduzindo genótipos taurinos continentais em cruzamentos com zebuínos e taurinos britânicos. Entretanto, quando se refere à EGC, representativa do acabamento da carcaça, os genótipos taurinos continentais são os mais deficitários, sendo os taurinos britânicos os que apresentam as maiores EGC à mensuração. Ratificando estes resultados, FELÍCIO (1999) afirma que as raças taurinas continentais, tardias (especialmente as "hipertróficas") e suas cruzas rendem maior porcentagem de cortes cárneos (desossados e com gordura bem aparada) do que as zebuínas, devido à maior proporção de gordura acumulada nessas últimas que são de acabamento relativamente mais precoce.

Buscando maior visualização dos efeitos dos genótipos sobre as características em estudo, foram realizadas simulações dos cruzamentos entre estes a partir das equações anteriores (Figura 2).

Para a simulação dos cruzamentos, foram utilizados valores de idade e peso vivo de 730 dias e $420 \mathrm{~kg}$, respectivamente, isso porque, segundo o Sistema Nacional de Tipificação de Carcaças Bovinas, sistema B-R-A-S-I-L (BRASIL, 1989), estes requisitos se enquadram na classificação de maior valor (B), além disso, denotam o que é chamado atualmente de "novilhos precoces".

Os genótipos puros zebuínos apresentaram AOL e EGC de $69,7 \mathrm{~cm}^{2}$ e 4,7mm, os taurinos britânicos, $70,3 \mathrm{~cm}^{2}$ e $8,1 \mathrm{~mm}$, e os taurinos continentais, $74,3 \mathrm{~cm}^{2} \mathrm{e}$ $3,7 \mathrm{~mm}$, considerando a média dos efeitos ambientais estudados. Estes resultados demonstram a dificuldade de se produzir o produto "novilho precoce", a partir de genótipos puros zebuínos e taurinos continentais, já que não atingem o mínimo de EGC de 5,0mm, recomendado para maior valorização das carcaças (USDA, 1997; CAMPOS et al., 2002), sendo deficientes em acabamento de carcaça. A forma de se contornar estes problemas é o aumento do aporte de nutrientes, confirmado pelas médias dos genótipos no sistema de alimentação confinado ou pelo abate em idades mais tardias.

Nos cruzamentos com os genótipos taurinos britânicos, a utilização de até $25 \%$ dos genótipos zebuínos ou taurinos continentais não causa prejuízo determinante no acabamento das carcaças, EGC, além de trazer inúmeros benefícios que convergem em importantes valores de heterose. No cruzamento entre zebuínos e taurinos continentais, os primeiros são os que promovem precocidade de acabamento aos animais cruzados, concordando com RESTLE et al. (2003), os quais compararam diferentes genótipos Charolês x Nelore.

\section{CONCLUSÕES}

A utilização da metanálise para comparação de atributos relacionados à qualidade de carcaça de bovinos de diferentes grupos genéticos, permitiu as seguintes conclusões: o país onde o animal foi criado,

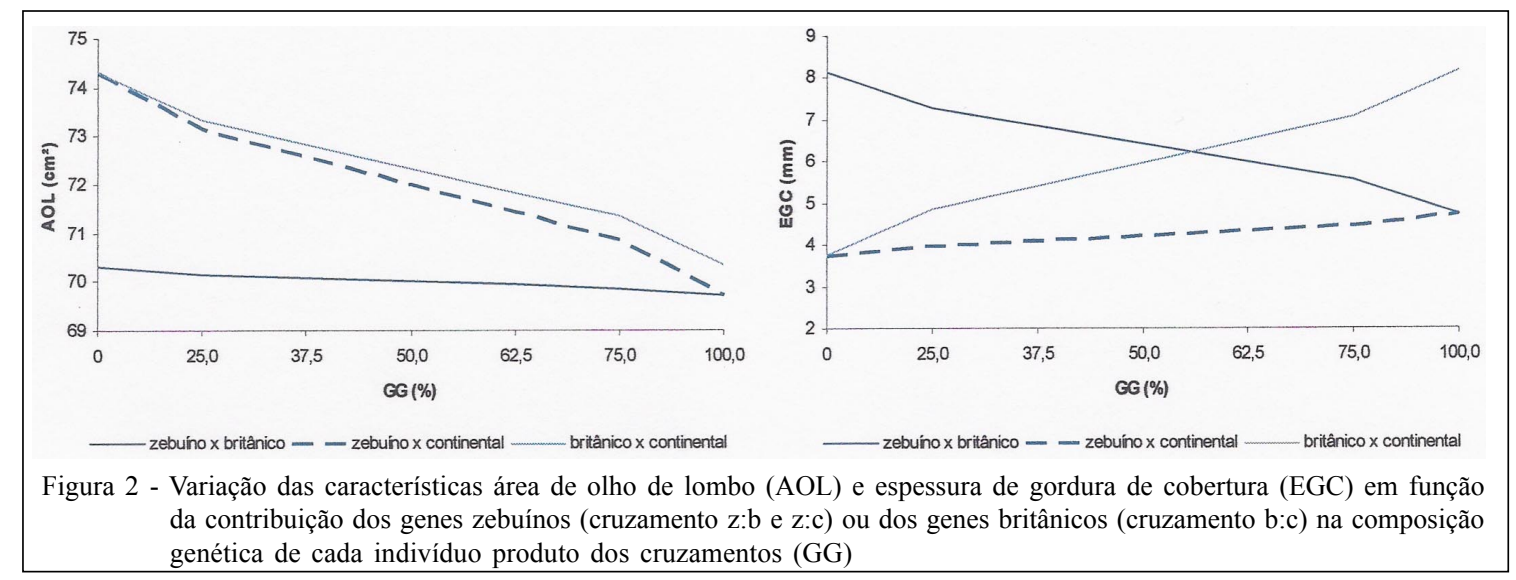

Ciência Rural, v.38, n.8, nov, 2008. 
a categoria animal, o sistema de alimentação em que o animal está inserido e a idade e o peso à mensuração das características afetam a variação da área de olho de lombo e espessura de gordura de cobertura e os genótipos taurinos continentais apresentaram os maiores valores de área de olho de lombo; os taurinos, de origem britânica, apresentaram os maiores valores para a espessura de gordura de cobertura, tendo os zebuínos apresentado valores intermediários para ambas as características.

\section{AGRADECIMENTO}

$\mathrm{O}$ autor agradeçe à Comissão de Aperfeiçoamento de Pessoal de Nível Superior (CAPES), pela concessão de bolsa.

\section{REFERÊNCIAS}

AGUILAR, I. ; BRIZOLARA, J.A. Relevamiento de la utilización de los cruzamientos en ganado de carne en establecimientos integrados a los grupos CREA del sector ganadero. 1995. 123f. Tesis (Grado) - Facultad de Agronomía. Universidad de la República.

ANDERSEN, B. B. et al. Comparison of ultrasonic equipment for describing beef carcass characteristics in live cattle (Report on a joint ultrasonic trial carried out in the U.K. and Denmark). Livestock Production Science, v.10, n.2, p.133-147, 1983.

BIDNER, T.D. et al. Influence of Brahman-derivative breeds and Angus on carcass traits, physical composition, and palatability. Journal of Animal Science, v.80, p.21262133, 2002 .

BRASIL. Ministério da Agricultura. Portaria n.612, 5 de outubro de 1989. Sistema nacional de tipificação de carcaças bovinas. Brasília, 1989 . $5 \mathrm{p}$.

CAMPOS, G. et al. Valorización objetiva de canales en función de atributos económicamente relevantes. In: SEMINARIO DE ATUALIZACIÓN TÉCNICA: CRUZAMIENTOS EN BOVINOS PARA CARNE. 2002, Tacuarembo. Anales... Tacuarembo, Uruguay: INIA, 2002. p.39-62.

CASAS, E.; CUNDIFF, L.V. Maternal grandsire, granddam, and sire breed effects on growth and carcass traits of crossbred cattle. Journal of Animal Science, v.81, p.904-911, 2003.

CATTANI, P. Perspectivas y avances de las forrajes conservados. In: CONGRESSO NACIONAL DE CONSERVACIÓN Y USO DE FORRAJES, 2., 2006, Rosario. Anales... Rosario, Argentina: IPCVA, 2006. p.7-13.

CROUSE, J.D. et al. C omparisons of Bos indicus and Bos taurus inheritance for carcass beef characteristics and meat palatability. Journal of Animal Science, v.67, n.9, p.26612668, 1989

CUNNINGHAM, J.G. Tratado de fisiologia veterinária. 3.ed. Rio de Janeiro: Guanabara Koogan, 2004. 579p.

EGGER, M.; SMITH, G.D. Meta-analysis: bias in location and selection of studies. British Journal of Medicine, v.61-66, 1998 .
FELÍCIO, P.E. de. Perspectivas para a tipificação de carcaça bovina. In: SIMPÓSIO INTERNACIONAL SOBRE TENDÊNCIAS E PERSPECTIVAS DA CADEIA PRODUTIVA DA CARNE BOVINA - SIMPOCARNE, 1999, São Paulo. Anais... São Paulo, SP: nl., 1999. np. Capturado em 12 dez. 2007. Online. Disponível em: http://www.fea.unicamp.br/deptos/ dta/carnes/files/ Simpocarne1.pdf

GALLINGER, M.M. et al. Meat quality of zebú Cross-breeds: sensory and mechanical evaluation. In: INTERNATIONAL CONGRESS OF MEAT SCIENCE AND TECHNOLOGY, 38 1992, Clermont-Ferrand. Proceedings... Clermont-Ferrand, France: American Meat Science Association, 1992. p.45-48.

GARNERO, A.V. et al. Parâmetros genéticos da taxa de maturação e do peso assintótico de fêmeas da raça Nelore. Arquivo Brasileiro de Medicina Veterinária e Zootecnia, v.57, n.5, p.652-662, 2005.

GREGORY, K.E. et al. Breed effects and retained heterosis for growth, carcass, and meat traits in advanced generations of composite populations of beef cattle. Journal of Animal Science, v.72, p.833-850, 1994.

HAUPTLI, L. et al. Comparação da adição de extratos vegetais e antimicrobianos sintéticos para leitões na creche através de metaanálise. Ciência Rural, v.37, n.4, p.1084-1090, 2007.

HEDGES, L.V.; OLKIN, I. Statistical methods for metaanalysis. London: Academic, 1985. 369p.

HEDRICK, H.B. Methods of estimating live animal and carcass composition. Journal of Animal Science, v.57, n.5, p.1316$1326,1983$.

KOCH, R.M. et al. Characterization of biological types of cattle cicle III: carcass composition, quality and palatability. Journal of Animal Science, v.54, n.1, p.35-45, 1982.

LEME, P.R. et al. Desempenho em confinamento e características de carcaça de bovinos machos de diferentes cruzamentos abatidos em três faixas de peso. Revista Brasileira de Zootecnia, v.29, n.6, supl.2, p.2347-2353, 2000.

MARSHALL, D.M. Breed differences and genetic parameters for body composition traits in beef cattle. Journal of Animal Science, v.72, p.2745-2755, 1994

MÜLLER, L. Normas para avaliação de carcaças e concurso de carcaças de novilhos. 2.ed. Santa Maria: UFSM, 1987. $31 \mathrm{p}$.

PERKINS, T.L. The use of real-time, linear-array ultrasound techniques to predict final carcass composition in beef catle. 1992. 117f. Tese (PhD) - Texas Tech University.

RESTLE, J. et al. Características de carcaça e da carne de vacas de descarte de diferentes genótipos charolês $\mathrm{x}$ nelore, terminadas em confinamento. Ciência Rural, v.33, n.2, p.345-350, 2003.

RIBEIRO, F.G. et al. Correlações entre medidas com ultra-som in vivo e diretamente na carcaça post mortem em bovinos. In: CONGRESSO DE ZOOTECNIA, 9., 1999, Portugal. Anais... Portugal: APEZ, 1999a. p.19. 
RIBEIRO, F.G. et al. Relação entre espessura de gordura e área de olho de lombo determinados in vivo e post mortem em bovinos jovens. In: REUNIÃO ANUAL DA SOCIEDADE BRASILEIRA DE ZOOTECNIA, 36. 1999, Porto Alegre. Anais... Porto Alegre: SBZ, 1999b, p.357.

ROUSE, G.H. et al. The accuracy of real-time ultrasound scans taken serially on small-, medium, and large-frame steers and bulls slaughtered at three endpoints. Beef \& Sheep Research Report. Ames: Iowa State University, 1992, p.14-19.

SANCHEZ, L. et al. Características de la canal y de la carne de machos procedentes del cruce de Rubia Gallega con Nelore. Archivos de Zootecnia, v.54, p.485-489, 2005.

SAS. Statistical Analysis Systems user's guide: Stat, Version 8 ed. Cary: SAS Institute, USA, 2001, 1464p.

SHAPIRO, S.S.; WILK, M.B. An analysis of variance test for normality (complete samples). Biometrika, v.52, p.591-611, 1965.

SILVA, S.L. et al. Correlações entre características de carcaça avaliadas por ultra-som e pós-abate em novilhos nelore, alimentados com altas proporções de concentrado. Revista Brasileira de Zootecnia, v.32, n.5, p.1236-1242, 2003.

ST-PIERRE, N.R. Meta-analysis of experimental data en the animal sciences. Revista Brasileira de Zootecnia, v.36, supl., p.343-358, 2007.

SUNDSTROM, B. et al. Application of crossbreeding to beef production opportunities, obstacles and challenges. In: WORLD CONGRESS ON GENETICS APPLIED TO LIVESTOCK PRODUCTION, 5, 1994, Guelph. Proceedings... Guelph, Ontario - Canada: nl., 1994. p.148-167.

URICK, J.J. et al. Maternal breed of sire effects on postweaning performance of heifer and steer progeny: postweaning growth and carcass characteristics. Journal of Animal Science, v.69, p.4377-4387, 1991

USDA. United states standards for grades of carcass beef. Washington D. C.: USDA. C.\&M.S. Livestock and Seed Division, $1997,16 \mathrm{p}$

WHEELER, T. L. et al. Mechanism associated with the variation in tenderness of meat from Brahman and Hereford cattle. Journal of Animal Science, v.68, p.4206-4220, 1990. 